\title{
Effect of oxygen on the biosynthesis of flavor compound 3-methylbutanal from leucine catabolism during batch culture in Carnobacterium maltaromaticum LMA 28
}

\author{
M. I. Afzal, ${ }^{*}$ K.-A. Boulahya, $†$ C. Paris, ${ }^{*}$ S. Delaunay, $† \ddagger$ and C. Cailliez-Grimal*1 \\ *Laboratoire d'ingénierie des biomolécules, Université de Lorraine, 2 avenue de la Forêt de Haye B.P. 172, \\ Vandoeuvre-lès-Nancy F-54505, France \\ †Laboratoire réactions et génie des procédés, Centre National de la Recherche Scientifique (CNRS), UPR 3349, \\ Vandoeuvre-lès-Nancy F-54505, France \\ ‡Laboratoire réactions et génie des procédés, Université de Lorraine, UPR 3349, Vandoeuvre-lès-Nancy, F-54505 France
}

\begin{abstract}
In this study, we demonstrated the effect of different dissolved oxygen concentrations (DOC) on cell growth and intracellular biosynthesis of 3-methylbutanal from leucine catabolism in Carnobacterium maltaromaticum LMA 28 during batch culture. The maximum specific growth rate was obtained in culture when DOC was controlled at $50 \%$ of air saturation. The specific consumption rates of glucose and specific production rates of lactate were higher at a DOC at 50 or $90 \%$ of air saturation. Carnobacterium maltaromaticum LMA 28 produced high quantities of 3 -methylbutanal and 3-methylbutanol during culture with DOC maintained at $90 \%$, suggesting that oxygen had a significant effect of the formation of these flavor compounds. This high formation of flavor compounds in an oxygen-rich environment was attributed to the simultaneous activation and stimulation of both $\alpha$-ketoacid decarboxylase (KADC) and $\alpha$-ketoacid dehydrogenase (KADH) pathways. Thus, intracellular biosynthesis of 3-methylbutanal can be controlled by modifying the DOC of the culture or food product during fermentation.
\end{abstract}

Key words: Carnobacterium maltaromaticum, flavor formation, L-leucine, metabolic pathway

\section{INTRODUCTION}

Carnobacterium maltaromaticum belongs to the lactic acid bacteria ( $\mathbf{L A B}$ ) group, and strains of this species have been reported in a variety of foods, including a few dairy products (Leisner et al., 2007; Afzal et al., 2010). Its presence in Brie, a protected designation of origin French surface mold-ripened soft cheese, was first reported in 1994 (Millière et al., 1994) and confirmed in 2007 (Cailliez-Grimal et al., 2007). Works published

Received August 27, 2012.

Accepted October 6, 2012.

${ }^{1}$ Corresponding author: catherine.cailliez@ensaia.inpl-nancy.fr in the past decade have shown that this species could be considered a nonstarter LAB (NSLAB), because it constitutes the main psychrotrophic LAB, is able to grow even at alkaline $\mathrm{pH}$ value, and reaches high concentrations $\left(10^{8}\right.$ to $\left.10^{9} \mathrm{cfu} / \mathrm{mL}\right)$ during cheese ripening (Cailliez-Grimal et al., 2007). Producing the malty or chocolate-like compound 3-methylbutanal from the catabolism of leucine is a general characteristic of this species (Miller et al., 1974; Leisner et al., 2007; Afzal et al., 2010). In the literature, it is reported that leucine can be converted into 3-methylbutanal by 1 of 2 metabolic pathways. A direct pathway implicating $\alpha$-ketoacid decarboxylase (KADC) enzyme or an indirect one with $\alpha$-ketoacid dehydrogenase (KADH) enzyme complex might be present in microorganisms, particularly those of the genera Lactococcus, Lactobacillus, Streptococcus, Enterococcus, and Staphylococcus (Yvon and Rijnen, 2001; Helinck et al., 2004; Smit et al., 2004; Figure 1).

Recently, we demonstrated the co-existence of both metabolic routes in C. maltaromaticum LMA 28 (Afzal et al., 2012). The functionality of these pathways is highly influenced by various factors including $\mathrm{pH}$, temperature, salt, and oxygen or redox environment (de la Plaza et al., 2004; Kieronczyk et al., 2006). Indeed, the presence of oxygen or high oxidation state results in increased production of 3-methylbutanal and 3-methylbutanol in Lactococcus lactis and Proteus vulgaris, respectively (Kieronczyk et al., 2006; Deetae et al., 2011). At the same time, enzyme activities involved in amino acid catabolism could be strongly controlled by the generation of oxidizing $\left(\mathrm{NAD}^{+}\right)$and reducing agents (NADH, $\mathrm{H}^{+}$) (Bourel et al., 2003; Pham et al., 2008). Anaerobic microorganisms convert pyruvate, resulting from glycolysis, into lactate through the activity of lactate dehydrogenase $(\mathbf{L D H})$, generating $\mathrm{NAD}^{+}$and ATP in the absence of oxygen. The activity of LDH has been shown to be dependent on the redox environment, exhibiting high specific activity in reducing conditions in Escherichia coli (Riondet et al., 


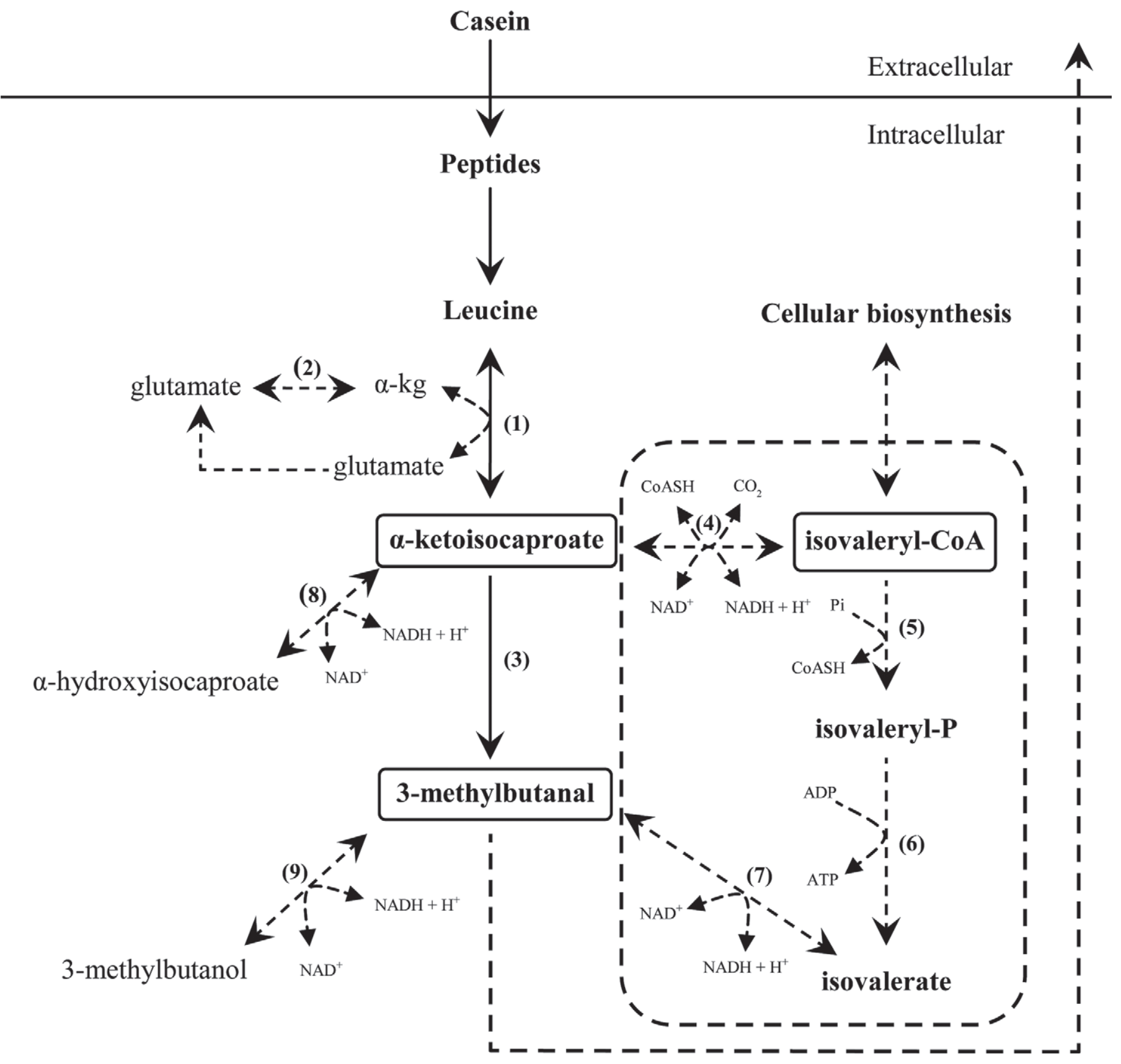

Figure 1. Intracellular metabolic pathways for the biosynthesis of 3-methylbutanal from catabolism of leucine by Carnobacterium maltaromaticum LMA 28 (Afzal et al., 2012). Enzymes of direct pathway (solid lines): (1) aminotransferase (AT), (2) glutamate dehydrogenase (GDH), (3) $\alpha$-ketoacid decarboxylase (KADC); enzymes of indirect pathway (dashed lines): (4) $\alpha$-ketoacid dehydrogenase (KADH), (5) phosphotransferase (PTA), (6) acylkinase (ACK), (7) aldehyde dehydrogenase (AldDH); other enzymes: (8) $\alpha$-hydroxyacid dehydrogenase (HADH), (9) alcohol dehydrogenase $(\mathrm{AlcDH})$.

2000). However, in aerobic microorganisms, pyruvate formation from glycolysis generates more $\mathrm{NADH}, \mathrm{H}^{+}$, and ATP in the presence of high dissolved oxygen concentration (DOC; Ardö, 2006; Pham et al., 2008). The presence of oxygen in culture medium, and consequently the redox environment, may have a dramatic effect on the control of amino acid catabolism. Previously, the increased production of 3-methylbutanal from leu- cine in Lactococcus lactis NCDO1867 under oxidative conditions was thought to be stimulated either by the increase in KADC activity or by substrate availability (Kieronczyk et al., 2006).

Carnobacteria are complex facultative anaerobic microorganisms, able to grow under low or high DOC, and able to produce lactic acid, ethanol, and $\mathrm{CO}_{2}$ from pyruvate. Therefore, the intracellular balance of $\mathrm{NAD}^{+}$ 
and $\mathrm{NADH}, \mathrm{H}^{+}$could play a major role in controlling the biosynthetic pathways of 3-methylbutanal. Because C. maltaromaticum LMA 28 possesses both metabolic pathways, our objective was to study how different DOC states in a chemically defined culture medium influence these pathways involved in the biosynthesis of the flavor compound 3-methylbutanal.

\section{MATERIALS AND METHODS}

\section{Strain and Medium Composition}

The bacterial strain studied, $C$. maltaromaticum LMA 28, was obtained from the culture collection of the Laboratoire de Microbiologie Alimentaire (Université de Lorraine, France). It was isolated from a mold-ripened soft cheese (Millière et al., 1994), and the strain was cultivated in a flask on a synthetic minimal medium called MCGC (Von der Osten et al., 1989), in which citrate was replaced by deferoxamine (Delaunay et al., 1999). The MCGC medium consisted of glucose $(40 \mathrm{~g} / \mathrm{L}), \mathrm{Na}_{2} \mathrm{HPO}_{4} \cdot 12 \mathrm{H}_{2} \mathrm{O}(45.5 \mathrm{~g} / \mathrm{L}), \mathrm{KH}-$ ${ }_{2} \mathrm{PO}_{4}(3.6 \mathrm{~g} / \mathrm{L}), \mathrm{NaCl}(1.2 \mathrm{~g} / \mathrm{L}),\left(\mathrm{NH}_{4}\right)_{2} \mathrm{SO}_{4}(4.8 \mathrm{~g} / \mathrm{L})$, $\mathrm{MgSO}_{4} \cdot 7 \mathrm{H}_{2} \mathrm{O}(0.4 \mathrm{~g} / \mathrm{L}), \mathrm{FeSO}_{4} \cdot 7 \mathrm{H}_{2} \mathrm{O}(40 \mathrm{mg} / \mathrm{L}), \mathrm{FeCl}_{3}$ $(4 \mathrm{mg} / \mathrm{L}), \mathrm{ZnSO}_{4} \cdot 7 \mathrm{H}_{2} \mathrm{O}(1 \mathrm{mg} / \mathrm{L}), \mathrm{CuCl}_{2} \cdot 2 \mathrm{H}_{2} \mathrm{O}(0.4$ $\mathrm{mg} / \mathrm{L}), \mathrm{MnSO}_{4} \cdot \mathrm{H}_{2} \mathrm{O}(4 \mathrm{mg} / \mathrm{L}),\left(\mathrm{NH}_{4}\right)_{6} \mathrm{Mo}_{7} \mathrm{O}_{24} \cdot 4 \mathrm{H}_{2} \mathrm{O}(0.2$ $\mathrm{mg} / \mathrm{L}), \mathrm{Na}_{2} \mathrm{~B}_{4} \mathrm{O}_{7} \cdot 10 \mathrm{H}_{2} \mathrm{O}(0.4 \mathrm{mg} / \mathrm{L}), \mathrm{CaCl}_{2}(84 \mathrm{mg} / \mathrm{L})$, biotin $(2 \mathrm{mg} / \mathrm{L})$, thiamine $(20 \mathrm{mg} / \mathrm{L})$, deferoxamine (3 $\mathrm{mg} / \mathrm{L})$, and glycine betaine $(2 \mathrm{~g} / \mathrm{L})$. This medium was modified by the supplementation of yeast extract (1 $\mathrm{g} / \mathrm{L}$ ) and its $\mathrm{pH}$ was adjusted to 6.8 . The bacterial strain was grown under aerobic conditions at $30^{\circ} \mathrm{C}$ and $200 \mathrm{rpm}$ in $500-\mathrm{mL}$ flasks for $40 \mathrm{~h}$. All chemicals were purchased from Sigma-Aldrich (St. Louis, MO).

Batch Culture in Bioreactor Under Different DOC. Carnobacterium maltaromaticum LMA 28 was cultured in a 5-L bioreactor (Applikon, Schiedam, the Netherlands) with a 1.5-L working volume. The MCGC medium for batch culture in the stirred bioreactor was the same as described earlier but modified by supplementation with leucine $(6.5 \mathrm{~g} / \mathrm{L})$. The volume of preculture used to inoculate the bioreactor was determined by aiming for an initial cellular concentration of approximately $10^{5} \mathrm{cfu} / \mathrm{mL}$. The $\mathrm{pH}$ was regulated to 6.8 by the addition of $\mathrm{NaOH}(12 N)$ or $\mathrm{HCl}(2 N)$ solution. The DOC was controlled by agitation, gas flow, and composition of the gas (air or $\mathrm{N}_{2}$ ). The gas flow varied from 60 to $100 \mathrm{~L} / \mathrm{h}$. To study the influence of oxygen on the biosynthesis of 3-methylbutanal from leucine catabolism by $C$. maltaromaticum LMA 28 , cultures were carried out under different DOC: in the absence of oxygen (0\%) or in the presence of oxygen (50 and $90 \%$ ) for $55 \mathrm{~h}$. The absence of oxygen in the culture medium (0\%) was achieved by continuous sparging of $\mathrm{N}_{2}$. Experiments were performed in duplicate, and samples were collected throughout culture to determine viable cell counts and concentrations of glucose, lactate, 3-methylbutanal, and 3-methylbutanol.

Viable Cell Number. Serial dilutions of samples were carried out in tryptone salt solution. A volume of $100 \mu \mathrm{L}$ was spread on agar plates (trypticase soy agar supplemented with yeast extract) with the help of a spiral plater (AES, Combourg, France). Agar plates were incubated at $30^{\circ} \mathrm{C}$, and bacterial counts $\left(\log _{10} \mathrm{cfu} /\right.$ $\mathrm{mL}$ ) were recorded after $48 \mathrm{~h}$.

Determination of Glucose and Lactate. After centrifugation of culture samples $(12,000 \times g$ for 5 min at $4^{\circ} \mathrm{C}$ ), the concentrations of glucose and lactate were determined enzymatically (glucose, Elitech, Paris, France; lactate, bioMérieux, Marcy l'Etoile, France).

Determination of 3-Methylbutanal and 3-Methylbutanol by Headspace Gas Chromatography. During culture of C. maltaromaticum LMA 28 under different DOC, samples $(1 \mathrm{~mL})$ were collected at different intervals and stored immediately in hermetic $10-\mathrm{mL}$ vials at $-25^{\circ} \mathrm{C}$ until analysis by headspace gas chromatography. A 1.0-mL headspace sample was injected (splitless) on the column after 2 min of incubation at $90^{\circ} \mathrm{C}$. The chromatograph (PR 2100, Périchrom, Saulxlès-Carthusian, France) was equipped with an injector with headspace (Headspace HT 300A, Périchrom) and a flame-ionization detector (Périchrom). The volatile compounds were separated using a capillary column of $30 \mathrm{~m} \times 0.25 \mathrm{~mm} \times 0.2 \mu \mathrm{m}$ and proportioned by integrator WINILAB III (Périchrom). The carrier gas was nitrogen at a constant flow of $2 \mathrm{~mL} / \mathrm{min}$. The temperature of the detector was maintained at $190^{\circ} \mathrm{C}$. The vials were pressurized for $30 \mathrm{~s}$ and injection lasted $1 \mathrm{~s}$. The adjustment of the quantity of product by the valve of open escape or "split" was 5:5. The oven temperature was initially kept at $45^{\circ} \mathrm{C}$ for $1 \mathrm{~min}$, increased by $5^{\circ} \mathrm{C} /$ min to $190^{\circ} \mathrm{C}$, and then maintained at $190^{\circ} \mathrm{C}$ for 12 min. Standard curves were carried out with solutions of 3 -methylbutanal and 3-methylbutanol with concentrations varying between 1 and $5,000 \mu M$.

Determination of $K A D C$ and $K A D H$ Enzyme Activities. One liter of MCGC medium was taken from each culture at the maximum production rate of 3-methylbutanal and 3-methylbutanol in sterile conditions. Cell-free extracts and protein contents, along with determination of KADC and $\mathrm{KADH}$, were measured as described previously (Afzal et al., 2012).

\section{RESULTS AND DISCUSSION}

\section{Bacterial Growth}

The growth of C. maltaromaticum LMA 28 during batch culture was influenced by the presence of oxygen 
(Figure 2). The viable cell concentration reached $6 \log _{10}$ $\mathrm{cfu} / \mathrm{mL}$ with a maximum specific growth rate of 0.02 $\mathrm{h}^{-1}$ in the absence of oxygen (Figure 2A), whereas the maximum viable cell concentration reached $7 \log _{10} \mathrm{cfu} /$ $\mathrm{mL}$ in the presence of oxygen, with maximum specific growth rates of 0.06 and $0.035 \mathrm{~h}^{-1}$ after $5 \mathrm{~h}$ of culture at a DOC of 50 and $90 \%$ of air saturation, respectively (Figure 2B and 2C).

Our results are consistent with previous findings showing that many LAB grow aerobically at a specific growth rate higher than that determined in the anaerobic state. For instance, Leuconostoc mesenteroides ssp. mesenteroides and Brochothrix thermosphacta ATCC 11509 showed high specific growth rates $(0.66$ and $0.50 \mathrm{~h}^{-1}$ ) under aerobic conditions compared with those achieved in the anaerobic states $(0.45$ and 0.20 $\mathrm{h}^{-1}$, respectively; Blickstad and Molin, 1984; Borch and Molin, 1989; Plihon et al., 1995).

The slightly different growth response of $C$. maltaromaticum LMA 28 during culture in the presence of oxygen might be due to its facultative anaerobic nature, consuming a substantial proportion of oxygen during the aerobic state, with increased growth yield and specific growth rates, as shown previously for Leuconostoc and Carnobacterium strains (Borch and Molin, 1989; Plihon et al., 1995).

\section{Influence of DOC on Glucose Consumption and Lactic Acid Production}

In the absence of oxygen, very slight consumption of glucose was noticed during the first $5 \mathrm{~h}$ of culture, with a specific consumption rate of $0.01 \mathrm{~g} / \log _{10}$ cfu per hour (Figure 3A). Moreover, no lactate was detected in the culture medium. The bacterial growth previously determined in this culture condition was thus probably due to the use of yeast extract instead of glucose as a carbon source. The maximum specific consumption rates of glucose were 0.03 and $0.04 \mathrm{~g} / \log _{10}$ cfu per hour with DOC of 50 and $90 \%$, respectively. In both culture conditions, $33 \mathrm{~g} / \mathrm{L}$ glucose remained in the medium at the end of the cultures, indicating that only a small amount of glucose was consumed (Figures 3B and 3C). The production of lactate was 1.8 and $0.9 \mathrm{~g} / \mathrm{L}$, with a specific production rate less than $0.01 \mathrm{~g} / \log _{10}$ cfu per hour in the presence of 50 or $90 \%$ air saturation, respectively (Figures $4 \mathrm{~A}$ and $4 \mathrm{~B}$ ).

The production of lactate was mainly due to $\mathrm{LDH}$, which remained active under the anaerobic environment, converting pyruvate into lactate. The acidifying activity of $C$. maltaromaticum LMA 28 was previously reported to be weak compared with that of commercial starter LAB (Edima et al., 2008). However, the capacity of Carnobacterium species to produce some lactic acid in the presence of oxygen was previously demonstrated. Various strains were shown to produce 0.21 to $0.28 \mathrm{~mol}$ of lactic acid per $1 \mathrm{~mol}$ of glucose during aerobic batch culture in yeast-peptone-thiamine growth medium (Borch and Molin, 1989).

\section{Effect of DOC on Biosynthesis of 3-Methylbutanal and 3-Methylbutanol}

The production of 3-methylbutanal and 3-methylbutanol was similar in the absence of oxygen and in presence of $50 \%$ air saturation, whereas a dramatic increase in the concentration of these 2 metabolites was determined with $90 \%$ air saturation. The maximum concentrations of 3-methylbutanal and 3-methylbutanol were, respectively, 190 and $320 \mu M$ in presence of $90 \%$ air saturation, whereas they were 120 to 130 and $100 \mu M$ in the absence of oxygen and with $50 \%$ air saturation, respectively (Figures 5 and 6 ). This finding suggests that DOC in the culture medium might have a significant effect on the biosynthesis of 3-methylbutanal and 3-methylbutanol in C. maltaromaticum LMA 28. Regarding the weak concentrations of 3-methylbutanal and 3-methylbutanol produced $(0.5 \mathrm{mM})$, no significant decrease in leucine concentration was determined (data not shown). This also suggests that leucine was catabolized only through 3-methylbutanal and 3-methylbutanol producing pathways. These results are in agreement with previous studies showing that such a change in the production of metabolites could be attributed to the modification of redox potential $\left(E_{\mathrm{h}}\right)$ of the culture medium by incorporation of oxygen or addition of oxidizing $\left(\mathrm{NAD}^{+}\right)$and reducing agents $\left(\mathrm{NADH}, \mathrm{H}^{+}\right)(\mathrm{Ki}-$ eronczyk et al., 2006; Deetae et al., 2011). However, no significant difference in the production of metabolites from leucine catabolism was observed in the presence or absence of oxygen by Carnobacterium piscicola strain 545 (Larrouture-Thiveyrat and Montel, 2003).

The biosynthesis of the flavor compounds 3-methylbutanal and 3-methylbutanol from leucine catabolism may arise via 2 metabolic routes: the direct (KADC) or the indirect (KADH) pathway, or both (Thierry et al., 2002; Helinck et al., 2004; Afzal et al., 2012). To determine the biosynthetic route responsible for synthesis of 3-methylbutanal and 3-methylbutanol in the absence or presence of oxygen, activities of KADC and KADH were determined during culture with $90 \%$ air saturation and during culture without oxygen. These determinations were performed when the specific production rates of 3-methylbutanal and 3-methylbutanol were maximal (Figures 5 and 6 ). The activity of KADC was 3 times higher with $90 \%$ air saturation $(3.95 \pm$ $1.48 \mathrm{nmol} / \mathrm{mg}$ per minute) than without oxygen (1.35 $\pm 0.49 \mathrm{nmol} / \mathrm{mg}$ per minute), suggesting that KADC 




Time (h)

Figure 2. Growth of Carnobacterium maltaromaticum LMA 28 in MCGC minimal medium, along with specific growth rates cultivated in bioreactors under different dissolved oxygen concentrations: (A) $0 \%$ oxygen, (B) $50 \%$ air saturation, and (C) 90\% air saturation. Curves with symbols $(\mathbf{\Lambda})$ represent cellular concentration, and curves without symbols represent the specific growth rate.

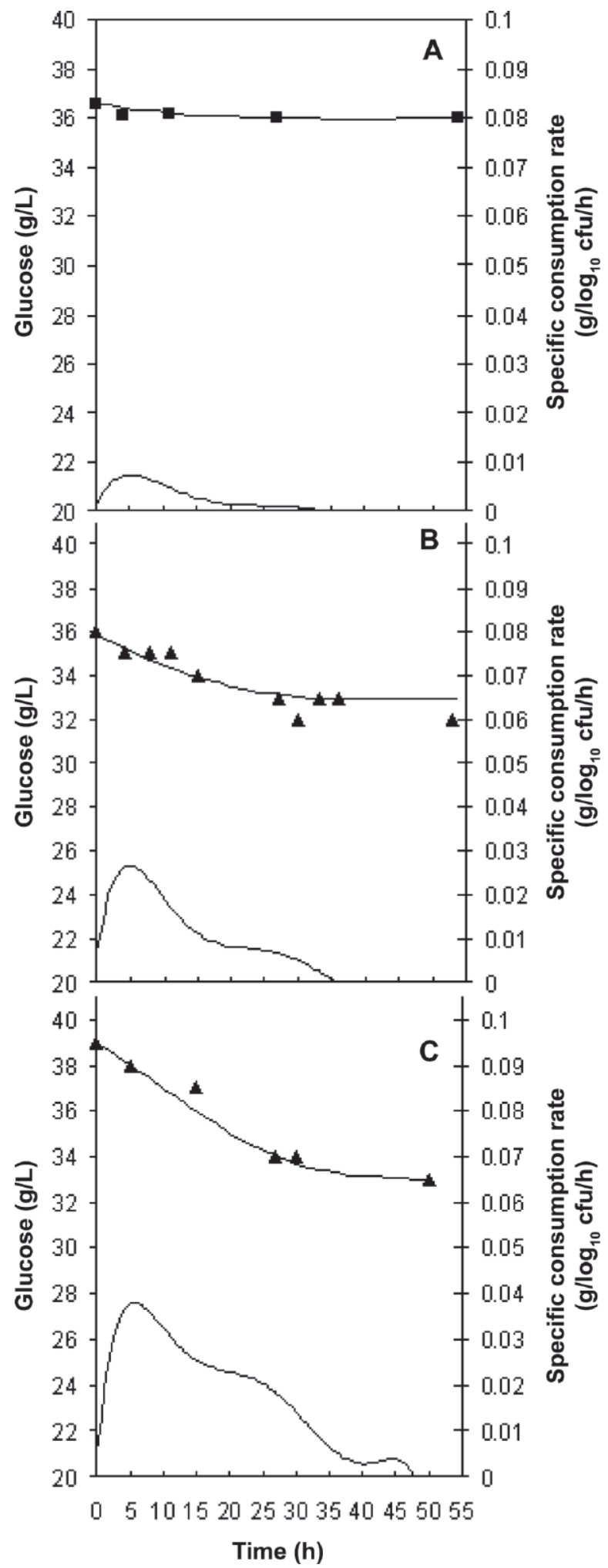

Figure 3. Consumption of glucose along with specific consumption rates during cultivation of Carnobacterium maltaromaticum LMA 28 in bioreactors under different dissolved oxygen concentrations: (A) $0 \%$ oxygen, (B) $50 \%$ air saturation, and (C) 90\% air saturation. Curves with symbols $(\boldsymbol{\Lambda})$ represent residual glucose concentration, and curves without symbols represent the specific rate of glucose consumption. 


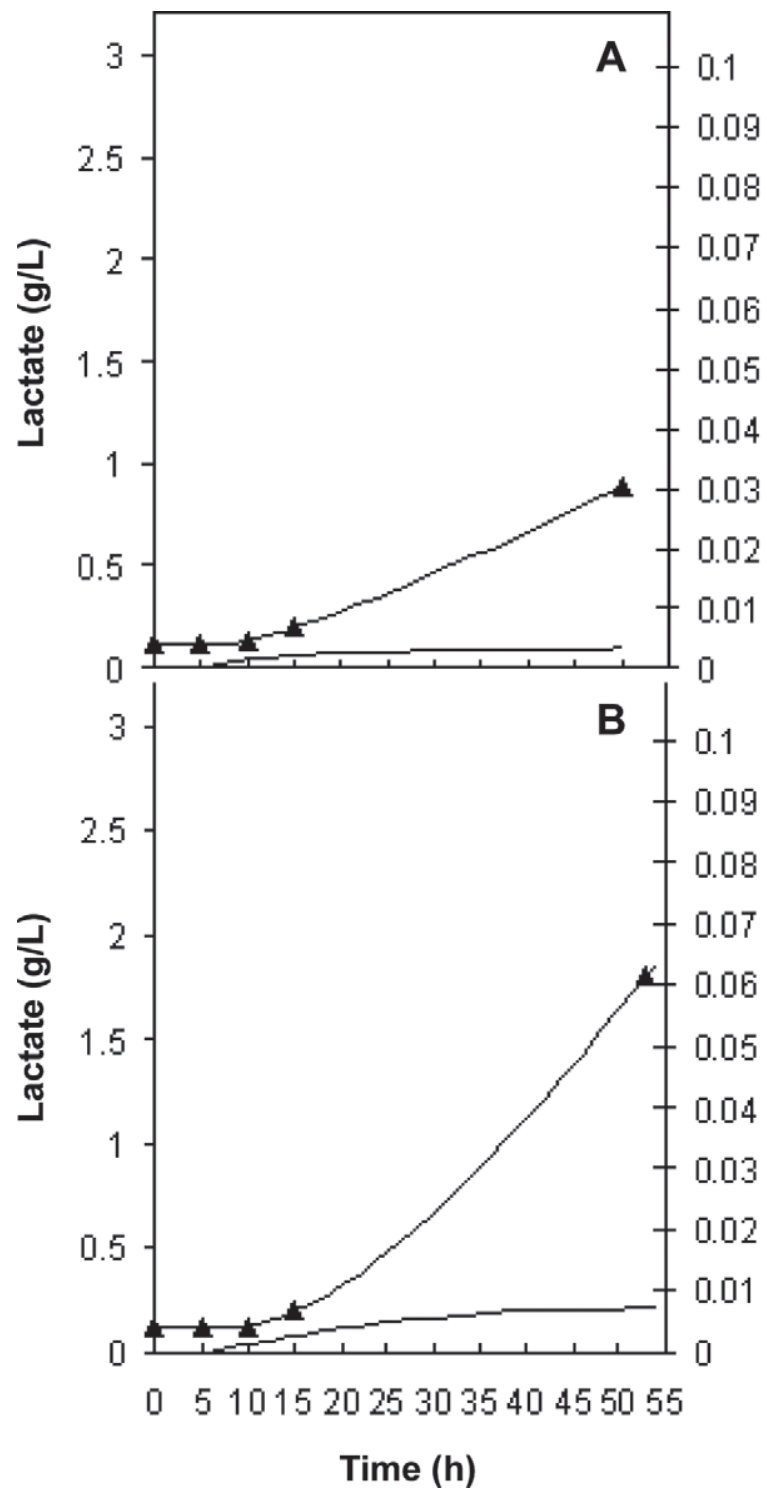

Figure 4. Production of lactate along with specific production rates during cultivation of Carnobacterium maltaromaticum LMA 28 in bioreactors under different dissolved oxygen concentrations: (A) 50\% air saturation and (B) 90\% air saturation. Curves with symbols (ム) represent lactate concentration, and curves without symbols represent the specific rate of lactate production.

might be more active under high DOC. The same tendency was observed for KADH activity: $1.67 \pm 0.01$ $\mathrm{nmol} / \mathrm{mg}$ per minute in the absence of oxygen and 3.5 $\pm 0.05 \mathrm{nmol} / \mathrm{mg}$ per minute with a DOC at $90 \%$ of air saturation. The presence of oxygen in the culture medium clearly induced an increase in the activity of both enzymes (which could be related to an increase in enzyme synthesis).

It was previously thought that extracellular redox potential might affect KADC and $\mathrm{KADH}$ activities. Under oxidative conditions, the production of 3-meth-

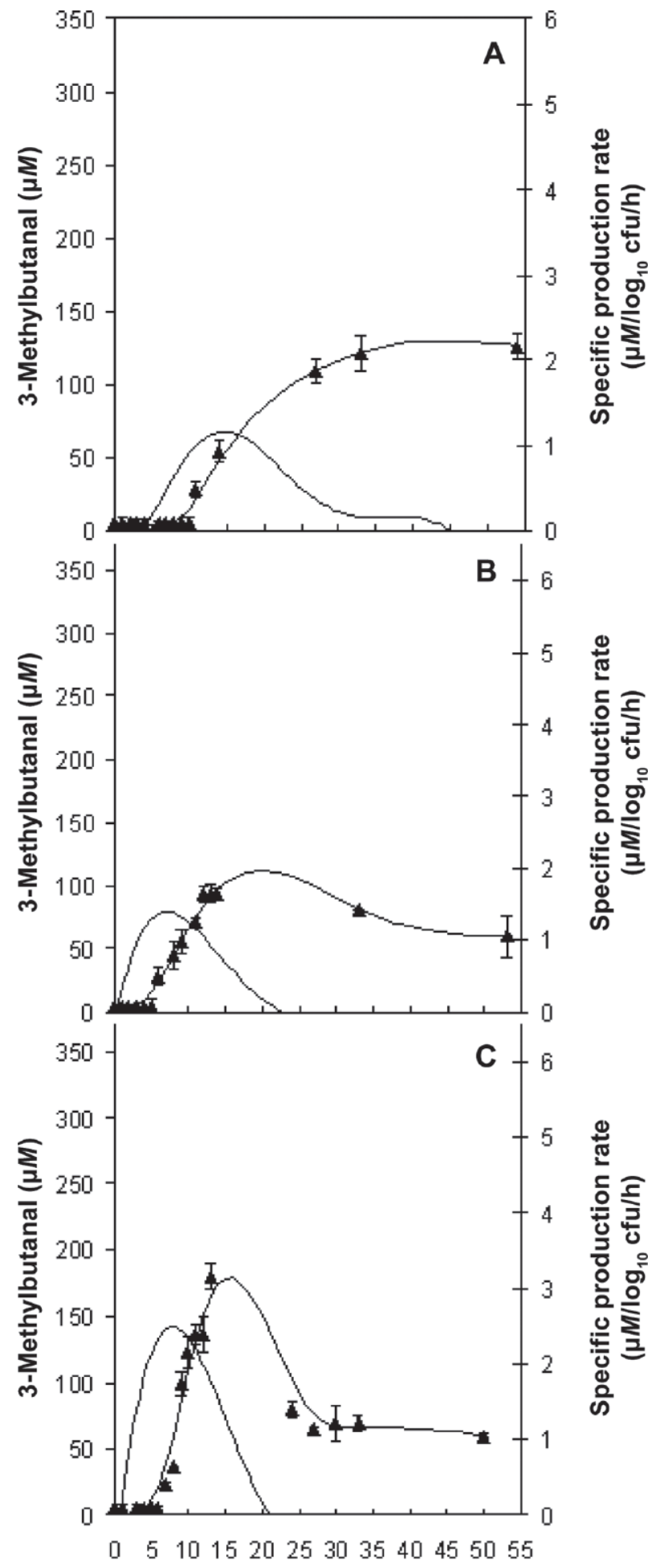

Time (h)

Figure 5. Biosynthesis of 3-methylbutanal along with specific production rates during cultivation of Carnobacterium maltaromaticum LMA 28 in bioreactors under different dissolved oxygen concentrations: (A) 0\% oxygen, (B) 50\% air saturation, and (C) 90\% air saturation. Curves with symbols $(\mathbf{\Lambda})$ represent 3-methylbutanal concentration, and curves without symbols represent the specific rate of 3-methylbutanal production. 


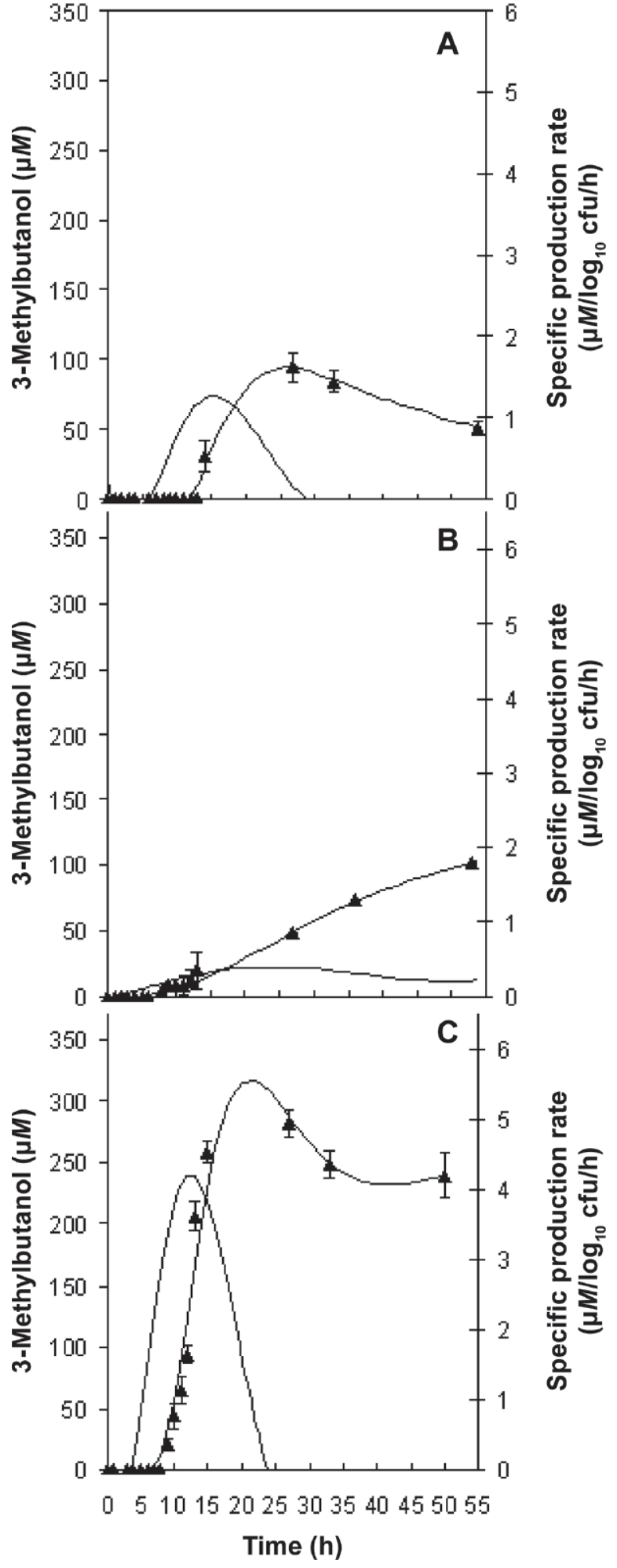

Figure 6. Biosynthesis of 3-methylbutanol along with specific production rates during cultivation of Carnobacterium maltaromaticum LMA 28 in bioreactors under different dissolved oxygen concentrations: (A) 0\% oxygen, (B) 50\% air saturation, and (C) 90\% air saturation. Curves with symbols $(\mathbf{\Lambda})$ represent 3-methylbutanol concentration, and curves without symbols represent the specific rate of 3-methylbutanol production. ylbutanal would mainly be due to KADC (Kieronczyk et al., 2006), whereas in reducing conditions, 3-methylbutanal would be synthesized mainly via the $\mathrm{KADH}$ pathway (Yvon and Rijnen, 2001). The determination of both KADC and KADH activities in the presence and absence of oxygen provided no answers concerning the respective roles of these 2 enzymes in C. maltaromaticum LMA 28, and further studies are required to clarify the role of these enzymes in synthesis of 3-methylbutanal. For instance, it has been shown that the ratio of $\mathrm{NAD}^{+}$to $\mathrm{NADH}, \mathrm{H}^{+}$affects pyruvate dehydrogenase activity, which is responsible for the oxidative decarboxylation of aromatic and branched-chain keto acids in Lactococcus lactis (Yvon and Rijnen, 2001). We could anticipate a similar effect on KADH enzyme activity.

\section{CONCLUSIONS}

In conclusion, DOC has a significant influence on the growth and biosynthesis of 3-methylbutanal and 3 -methylbutanol in C. maltaromaticum LMA 28. The highest DOC tested resulted in increased production of both flavor compounds. This increase might be due to stimulation of both KADC and KADH pathways as revealed by the determination of enzyme activities under different DOC states. To control the desired formation of 3-methylbutanal by C. maltaromaticum LMA 28 in food applications, external parameters, such as oxygen or redox potential, should be considered and controlled

\section{ACKNOWLEDGMENTS}

We thank Myriam Michel (Laboratoire d'ingénierie des biomolécules, Nancy, France) for her excellent technical assistance.

\section{REFERENCES}

Afzal, M. I., S. Delaunay, C. Paris, F. Borges, A. M. Revol-Junelles, and C. Cailliez-Grimal. 2012. Identification of metabolic pathways involved in the biosynthesis of flavor compound 3-methylbutanal from leucine catabolism by Carnobacterium maltaromaticum LMA 28. Int. J. Food Microbiol. 157:332-339.

Afzal, M. I., T. Jacquet, S. Delaunay, F. Borges, J. B. Milliere, A. M. Revol-Junelles, and C. Cailliez-Grimal. 2010. Carnobacterium maltaromaticum: Identification, isolation tools, ecology and technological aspects in dairy products. Food Microbiol. 27:573-579.

Ardö, Y. 2006. Flavour formation by amino acid catabolism. Biotechnol. Adv. 24:238-242

Blickstad, E., and G. Molin. 1984. Growth and end-product formation in fermenter cultures of Brochothrix thermosphacta ATCC 11509T and two psychrotrophic Lactobacillus spp. in different gaseous atmospheres. J. Appl. Bacteriol. 57:213-220.

Borch, E., and G. Molin. 1989. The aerobic growth and product formation of Lactobacillus, Leuconostoc, Brochothrix, and Carnobacterium in batch cultures. Appl. Microbiol. 30:81-88.

Bourel, G., S. Henini, C. Divies, and D. Garmyn. 2003. The response of Leuconostoc mesenteroides to low external oxidoreduction potential generated by hydrogen gas. J. Appl. Microbiol. 94:280-288. 
Cailliez-Grimal, C., H. C. Edima, A. M. Revol-Junelles, and J. B. Millière. 2007. Short communication: Carnobacterium maltaromaticum: The only Carnobacterium species in French ripened soft cheeses as revealed by polymerase chain reaction detection. J. Dairy Sci. 90:1133-1138.

de la Plaza, M., P. Fernández de Palencia, C. Peláez, and T. Requena. 2004. Biochemical and molecular characterization of $\alpha$-ketoisovalerate decarboxylase, an enzyme involved in the formation of aldehydes from amino acids by Lactococcus lactis. FEMS Microbiol. Lett. 238:367-374.

Deetae, P., A. Saint-Eve, H. E. Spinnler, and S. Helinck. 2011. Critical effect of oxygen on aroma compound production by Proteus vulgaris. Food Chem. 126:134-139.

Delaunay, S., P. Gourdon, P. Lapujade, E. Mailly, E. Oriol, J. M. Engasser, N. D. Lindley, and J. L. Goergen. 1999. An improved temperature-triggered process for glutamate production with $\mathrm{Co}_{\mathrm{O}}$ rynebacterium glutamicum. Enzyme Microb. Technol. 25:762-768.

Edima, H. C., C. Cailliez-Grimal, A. M. Revol-Junelles, E. Rondags, and J. B. Millière. 2008. Short communication: Impact of $\mathrm{pH}$ and temperature on the acidifying activity of Carnobacterium maltaromaticum. J. Dairy Sci. 91:3806-3813.

Helinck, S., D. Le Bars, D. Moreau, and M. Yvon. 2004. Ability of thermophilic lactic acid bacteria to produce aroma compounds from amino acids. Appl. Environ. Microbiol. 70:3855-3861.

Kieronczyk, A., R. Cachon, G. Feron, and M. Yvon. 2006. Addition of oxidizing or reducing agents to the reaction medium influences amino acid conversion to aroma compounds by Lactococcus lactis. J. Appl. Microbiol. 101:1114-1122.

Larrouture-Thiveyrat, C., and M. C. Montel. 2003. Effects of environmental factors on leucine catabolism by Carnobacterium piscicola. Int. J. Food Microbiol. 81:177-184.

Leisner, J. J., B. G. Laursen, H. Prevost, D. Drider, and P. Dalgaard. 2007. Carnobacterium: Positive and negative effects in the environment and in foods. FEMS Microbiol. Rev. 31:592-613.
Miller, A., M. E. Morgan, and L. M. Libbey. 1974. Lactobacillus maltaromicus, a new species producing a malty aroma. Int. J. Syst. Bacteriol. 24:346-354.

Millière, J. B., M. Michel, F. Mathieu, and G. Lefebvre. 1994. Presence of Carnobacterium spp. in French surface mould-ripened softcheese. J. Appl. Microbiol. 76:264-269.

Pham, T. H., G. Mauvais, C. Vergoignan, J. De Coninck, F. Dumont, J. Lherminier, R. Cachon, and G. Feron. 2008. Gaseous environments modify physiology in the brewing yeast Saccharomyces cerevisiae during batch alcoholic fermentation. J. Appl. Microbiol. 105:858-874.

Plihon, F., P. Taillandier, and P. Strehaiano. 1995. Oxygen effect on batch cultures of Leuconostoc mesenteroides: Relationship between oxygen uptake, growth and end-products. Appl. Microbiol. Biotechnol. 43:117-122.

Riondet, C., R. Cachon, Y. Wache, G. Alcaraz, and C. Divies. 2000. Extracellular oxidoreduction potential modifies carbon and electron flow in Escherichia coli. J. Bacteriol. 182:620-626.

Smit, B. A., W. J. Engels, J. T. Wouters, and G. Smit. 2004. Diversity of L-leucine catabolism in various microorganisms involved in dairy fermentations, and identification of the rate-controlling step in the formation of the potent flavour component 3-methylbutanal. Appl. Microbiol. Biotechnol. 64:396-402.

Thierry, A., M. B. Maillard, and M. Yvon. 2002. Conversion of Lleucine to isovaleric acid by Propionibacterium freudenreichii TL 34 and ITGP23. Appl. Environ. Microbiol. 68:608-615.

Von der Osten, C. H., C. Gioannetti, and A. J. Sinskey. 1989. Design of a defined medium for growth of Corynebacterium glutamicum in which citrate facilitates iron uptake. Biotechnol. Lett. 111:11-16.

Yvon, M., and L. Rijnen. 2001. Cheese flavour formation by amino acid catabolism. Int. Dairy J. 11:185-201. 